\title{
An infiltrating rheumatoid nodule?
}

\author{
A H Isdale, P S Helliwell
}

\begin{abstract}
The case is presented of a patient with mild rheumatoid arthritis who developed an enlarged painful tissue mass in the left lower leg. Although the history, clinical, and imaging appearances suggested that it was a soft tissue sarcoma, a biopsy specimen showed histological features consistent with either a rheumatoid nodule or subcutaneous granuloma annulare.
\end{abstract}

The differential diagnosis of soft tissue lesions of the anterior compartment of the leg include malignant tumours such as sarcomas and benign lesions such as subcutaneous granuloma annulare. Although the histological differentiation of subcutaneous granuloma annulare from rheumatoid nodules can be exceedingly difficult, rheumatoid nodules do not commonly occur at this site except in the childhood disease benign rheumatoid nodulosis. In addition rheumatoid nodules are usually seen in patients with severe seropositive rheumatoid arthritis (RA).

\section{Case report}

A 57 year old woman developed classical acute onset RA at the age of 36 years with a positive rheumatoid factor of $1 / 64$. She did well for four years while receiving symptomatic treatment alone but then required disease modifying drugs and treatment with penicillamine was started in 1973. She responded well to this and remained well until May 1987, when she experienced pain in the left lower leg which became worse at night. At this time a slight tenderness in the left anterior tibial compartment was noted. No lesion was seen and no neurological deficit was found. A full blood count was normal and her plasma viscosity was $1.74 \mathrm{cP}$. She was at this time RA latex negative and negative for antinuclear factor. By December 1987 an associated swelling was noticed at the site of tenderness and the possibility of pretibial myxoedema was suggested (thyroid function tests were normal). By April 1988 she had a palpable firm tender swelling on the anterolateral aspect of the left leg which appeared to be attached to underlying tissues. A radiograph was normal. Bone scintigraphy showed marked soft tissue uptake in the middle third of the anterolateral aspect of the left calf. There was no bony involvement. Before taking a biopsy specimen a computed tomography scan was performed which showed diffuse infiltration of the anterior compartment of the left lower leg over a fairly wide extent by a heterogeneous tumour, together with oedema of the subcutaneous fat (fig 1). This was thought to be a soft tissue sarcoma. A biopsy specimen was taken for assessment but this showed a granulomatous lesion with central fibrinoid necrosis surrounded by palisaded histiocytes with occasional multinucleate giant cells within an area of fibrosis with some lymphocytic infiltration (fig 2). No acid fast bacillae or fungal elements were identified. The pathologist thought this was a rheumatoid nodule in view of the patient's disease but it was also compatible with subcutaneous granuloma annulare.

Radiographs of the hands and feet at the time of writing show marked juxta-articular osteoporosis but no erosions. Rheumatoid factor is positive in a titre of $1 / 80$. A soft tissue mass remains palpable in the leg but no other nodules are palpable.

\section{Discussion}

Rheumatoid nodules occur in about $20-30 \%$ of patients with RA, especially in those patients with positive rheumatoid factor, more severe disease, and other extra-articular manifestations. Although rheumatoid nodules have been reported in a number of sites, their occurrence over the pretibial area has not been specifically reported. In contrast, in benign rheumatoid nodulosis of childhood this site is sometimes affected. In this disease the nodules tend to be multiple, painless, and generally regress, although there may be subsequent recurrences. There is no associated arthritis or connective tissue disease and the prognosis is excellent. ${ }^{1}$ Although clinically similar nodules may occur in rheumatic fever and juvenile chronic arthritis they can be distinguished histologically from benign rheumatoid nodulosis and the nodules of adult onset RA. ${ }^{23}$

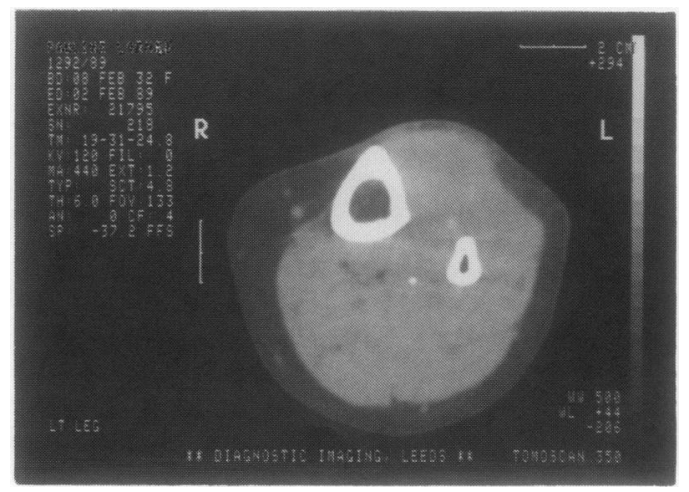

Figure 1 Computed tomography scan of the left lower leg. 

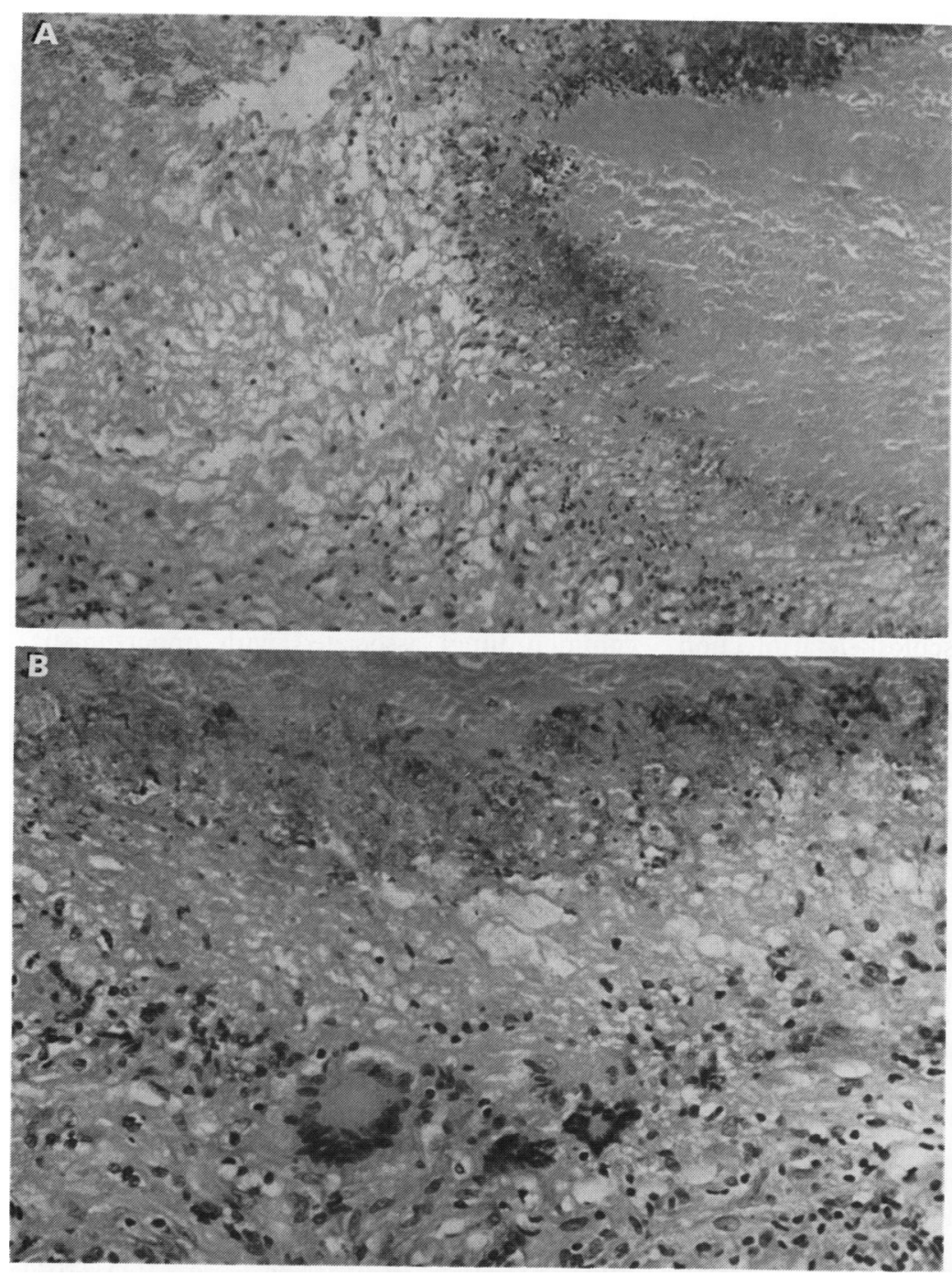

Figure 2 Part of a non-caseating granuloma lying within an area of fibrosis with some lymphocytic infiltrate. (A) Central fibrinoid necrosis surrounded by palisaded histiocytes and (B) higher magnification shows the multinucleate giant cell (haematoxylin-eosin stain).

Of particular interest is the almost identical pathological appearance of rheumatoid nodules (both benign and associated with RA) and subcutaneous granuloma annulare. ${ }^{4}$ The terminology is confusing and subcutaneous granuloma annulare, rheumatoid nodulosis, pseudorheumatoid nodule, benign rheumatoid nodule, and rheumatoid-like nodule appear to be used interchangeably, reflecting their similar histological appearances. In all respects the clinical features of subcutaneous granuloma annulare are identical with those of benign rheumatoid nodulosis of childhood. Subcutaneous granuloma annulare, however, almost certainly occur in adults and reports of 'rheumatoid nodules' occurring in seronegative patients who do not have, nor develop, $\mathrm{RA}^{56}$ are probably representative of adult subcutaneous granuloma annulare. This may even be true of some reports of apparent rheumatoid nodules occurring in seropositive patients without arthritis. ${ }^{78}$

Patterson ${ }^{9}$ looked more closely at the histology of subcutaneous granuloma annulare and rheu- matoid nodules and he was able on careful histological examination using selected histochemical and immunohistochemical techniques, to differentiate these lesions. He took from the pathology files of the Medical College of Virginia details of all patients diagnosed as having rheumatoid nodules, subcutaneous granuloma annulare, deep granuloma annulare, necrobiotic granuloma, and rheumatoid-like nodules and divided these, where possible, into either rheumatoid nodules or subcutaneous granuloma annulare according to the patients notes. Nine patients with subcutaneous granuloma annulare (benign rheumatoid nodules) and 12 patients with rheumatoid nodules were identified. The necrobiosis in rheumatoid nodules has a homogenous eosinophilic appearance, whereas in subcutaneous granuloma annulare there is a mucinous oedematous appearance. Patterson did not see giant cells in subcutaneous granuloma annulare and lymphocytes in the surrounding stroma were less prominent. Vascular changes were identical but there was usually only mild fibrosis in subcutaneous granuloma annulare compared with rheumatoid nodules. The staining patterns of Alcian blue (stains mucin), trichrome and Putts's (stains fibrin) additionally differentiated the two lesions although Alcian blue was the most specific (positive in one of 12 patients with rheumatoid nodules and all patients with subcutaneous granuloma annulare). This study, however, has not been validated by other pathologists and no blinded appraisal was made.

In the patient reported here, the site of the lesion, the absence of severe rheumatoid disease, and the low titre of rheumatoid factor is unusual for rheumatoid nodules and would be more in keeping with subcutaneous granuloma annulare. The presence of giant cells, however, together with fibrosis with lymphocytic infiltrates in the histological section would indicate, according to Patterson's appraisal, that the diagnosis of rheumatoid nodules is correct. It could be argued that long term penicillamine treatment in this patient has prevented the progression of her RA in other respects.

We thank Dr J Wyatt for preparing the figures.

1 Simons F E R, Schaller J G. Benign rheumatoid nodules. Pediatrics 1975; 56: 29-33.

2 Lowney E D, Simons H M. Rheumatoid nodules of the skin. Their significance as an isolated finding. Arch Dermatol $1963 ; 88: 853-8$.

3 Bywaters E G L. On two papers dealing with nodules [editorial]. F Rheumatol 1979; 6: 243-6.

4 Fitzpatrick T B, et al, eds. Dermatology in general medicine. 3rd ed. New York: McGraw Hill, 1971: 1859-70.

5 Burton J L. Granuloma annulave, rheumatoid nodules and necrobiosis lipoidica. Br $\mathcal{F}$ Dermatol 1977; 97 (suppl 15): $52-4$

6 Brown M M, Hadler N M, Sams W M, Wilson J, Snyderman R. Rheumatoid nodulosis. Sporadic and familial diseases. F Rheumatol 1979; 6: 286-92.

7 Ginsberg M H, Grenant H K, Yu T F, McCarty D J Rheumatoid nodulosis. An unusual variant of rheumatoid Rheumatoid nodulosis. An unusual variant
disease. Arthritis Rheum 1975; 18: 49-58.

8 Belin D C, Abeles M, Weinstein A. Rheumatoid markers in the absence of arthritis. I Rheumatol 1979; 6: 293-9.

9 Patterson J W. Rheumatoid nodule and subcutaneous granuloma annulare. Am $\mathcal{F}$ Dermatopathol 1988; 10: 1-8. 